\title{
EFFECT OF BUXUS CHINENSIS, DIPEL 2X AND NUCLEAR POLYHEDROSIS VIRUS (NPV) MIXTURE ON SOME BIOLOGICAL ASPECTS OF COTTON LEAFWORM SPODOPTERA LITTORALIS
}

\author{
ABD-ELZAHER, S. MAHMOUD and ABEER. M. MOHAMMAD
}

Plant Protection Research Institute, ARC, Giza, Egypt

(Manuscript received 26 September 2013)

\begin{abstract}
Effects of Buxus chinensis extract, Bacillus thuringiensis and nuclear polyhedrosis virus (NPV) mixture on certain biological aspects of Spodoptera littoralis were studied against the $2^{\text {nd }}$ and $4^{\text {th }}$ larval instars of $S$. littoralis. The mixture of the three compounds was the most toxic against the $2^{\text {nd }}$ and $4^{\text {th }}$ instar larvae, where it caused high percentage of mortality, while $B$. thuringiensis (Dipel $2 \mathrm{x}$ ) and nuclear polyhedrosis virus (NPV) mixture induced a slight effect against the $2^{\text {nd }}$ and $4^{\text {th }}$ instars. Regarding the ethanolic extract of Buxus chinensis leaves, it was more effective than $B$. thuringiensis (Dipel 2x) or nuclear polyhedrosis virus (NPV) in respect of pupal mortality and larval, pupal and adult duration. This plant extract also reduced the fecundity and fertility of females emerged from the treated larvae of $S$. littoralis.
\end{abstract}

Key words: Spodoptera littoralis, Buxus chinensis, Dipel $2 x, N P V$

\section{INTRODUCTION}

The cotton leafworm, Spodoptera littoralis (Boisd) is one of the most serious insect pests of many different Egyptian crops, it attacks and damages several parts of its host plants. Many researchers used different methods in order to control this pest (Chanda and Chakravorty 1993, Antonious and Rizk 1994, Abo El-Ghar , 1994, Schmidt et al., 1997, Ismail et al., 1999 , Marei et al., 2009 and Abd El-Ghany et al., 2012). Aim of the present work is to evaluate the effect of Buxus chinensis, B. thuringiensis (Dipel $2 \mathrm{x}$ ) and nuclear polyhedrosis virus (NPV) mixture on certain biological aspects of Spodoptera littoralis.

\section{MATERIALS AND METHODS}

\section{Insect culture.}

Laboratory strains of Spodoptera littoralis (Boisd) were obtained from Plant Protection Res. Institute, ARC, Dokki, Giza, Egypt. It was reared on Castor bean leaves according to El-Defrawi et al., (1964). The culture was maintained at $28^{\circ} \mathrm{C} \pm 2$ \& $55 \% \mathrm{RH}$, away from insecticides exposure for more than 10 generations. 


\section{Preparation of plant extracts .}

Buxus chinensis leaves were left to dry at room temperature of $28 \pm 2{ }^{\circ} \mathrm{C}$ for one week. The dried leaves were grounded to fine powder and extracted consecutively in a Soxhlet apparatus using ethanol solvent. Crude extracts were dried and filtered over anhydrous sodium sulphate and were subjected to remove the solvent used in the extraction. All the crude extracts obtained were kept in the refrigerator until use.

\section{Bioassay of compounds on Spodoptera littoralis.}

The tested compounds were used at concentrations of 4 ppm, $300 \mathrm{~g} / 400 \mathrm{~L}$ water and $5 \times 10^{12}$ /BIP $\mathrm{mL}$ for Buxus chinensis, B. thuringiensis (Dipel $2 \mathrm{x}$ ) and nuclear polyhedrosis virus (NPV), respectively. For bioassay experiment, leaf dipping technique was used. Castor bean leaves were dipped in the different concentrations of tested compounds. Then treated leaves were left in air dry. After leaves drying they were offered for the $2^{\text {nd }}$ and $4^{\text {th }}$ instars to fed them for $24 \mathrm{hrs}$.

For control, the fresh castor-bean leaves were immersed for five seconds in $10 \mathrm{ml}$ of each used insecticide concentration. The treated castor bean leaves were used as a food for both $2^{\text {nd }}$ and $4^{\text {th }}$ instars of $S$. littoralis according to the method of (Nakanishi, 1977) with modification. Two sets of experiments were carried out and the toxicity of each concentration was determined according to POLOPC (Leora Software, 1994). The effects on the insect development by the treatment with the mixture of Buxus chinensis extract, B. thuringiensis and nuclear polyhedrosis virus (NPV) at different concentrations ( offered for 72 -hrs to one hundred larvae of $2^{\text {nd }}$ and $4^{\text {th }}$ instars) were recorded.

After that untreated leaves were introduced daily to larvae until pupation 24-hrs after treatment the insects were examined daily and all biological parameters of the insect survivors (Mortalities as larvae, pupae and adult) were counted and recorded as percentage in relation to the total number of insects of the preceding stage. The biological efficacy of the different used compounds were calculated according to Vagras and Sehnal , 1973.

\section{Data analysis.}

Data were subjected to the probit analysis (Finney, 1971). When it is necessary the control mortality was adjusted by using Abbott's formula (Abbott, 1925).

\section{RESULTS AND DISCUSSION}

\section{1- Effect of ethanolic Buxus chinensis leaf extracts on $2^{\text {nd }}$ and $4^{\text {th }}$ instar larvae.}

Results presented in Table (1) show the effect of ethanolic Buxus chinensis leaf extracts on $2^{\text {nd }}$ and $4^{\text {th }}$ instar larvae of Spodoptera littoralis fed on castor bean leaves, The mean percentage of larval mortality was $82,60,54,45,35$ and $15 \%$ 
for $2^{\text {nd }}$ instars, while it was $27.5,20,16.5,15.5,13$ and $9.5 \%$ for the $4^{\text {th }}$ instars at concentrations of $4,2,1,0.5,0.25$ and 0.125 ppm of ethanolic Buxus chinensis leaf extracts, respectively. Data in Table (1) showed a significant effect on the larval duration of $2^{\text {nd }}$ and $4^{\text {th }}$ instar larvae, was obtained after treatment with different concentrations of ethanolic Buxus chinensis leaf extract. At concentrations of $4 \mathrm{ppm}$, it averaged 12.32 and 4.72 days for the $2^{\text {nd }}$ and the $4^{\text {th }}$ ones, respectively. The mean percentage of pupal mortality was 15 and $10 \%$ for the $2^{\text {nd }}$ and the $4^{\text {th }}$ instar as compared to zero mortality for the control treatment.

Regarding pupal duration, data recorded in Table (1), show that there was a significant effect on pupal duration resulted from treated $2^{\text {nd }}$ and $4^{\text {th }}$ instar larvae with ethanolic leaf extract at $4 \mathrm{ppm}$ concentration of Buxus chinensis while, the pupal duration was 7.14 and 7.11 days for the $2^{\text {nd }}$ and the $4^{\text {th }}$ treated larvae, respectively. As it was 9.8 and 8.5 days for the two untreated larval instars, respectively.

Taking into considration adult stage, resulted from treated $2^{\text {nd }}$ and $4^{\text {th }}$ larvae of Spodoptera littoralis, the mean percentage of emerged moths, fecundity and hatchability were greatly affected as presented in Table (1). The mean percentage of emerged moths was decreased up to $15 \%$ for $2^{\text {nd }}$ instars while it was $69 \%$ for the $4^{\text {th }}$ instars at the concentration of 4 ppm, respectively, compared to $100 \%$ emerged moths for control.

The concentration of the tested plant extracts played an important role in this respect. Concurrently, the higher the concentration of plant extracts the higher was the larval mortality and vice versa.

\section{2- Effect of mixture of Buxus chinensis leaf extracts, B. thuringiensis and nuclear polyhedrosis virus (NPV)}

When the $2^{\text {nd }}$ and the $4^{\text {th }}$ instars of Spodoptera littoralis were fed on castor oil leaves treated with the mixture of Buxus chinensis, B. thuringiensis and nuclear polyhedrosis virus (NPV), it was effective on the insect development (Table 2 ). From the data recorded in Table 2, it was observed that the mean percentage of larval mortality was increased by increasing concentrations. About $99 \%$ mortality was obtained at the higher concentrations for the $2^{\text {nd }}$ instars but it was $51 \%$ for the $4^{\text {th }}$ instars with mixtures of Buxus chinensis, B. thuringiensis and nuclear polyhedrosis virus (NPV), compared to no mortality for control larvae. 
Table 1. The effect of ethanolic Buxus. Chinensis leaf extract on $2^{\text {nd }}$ and $4^{\text {th }}$ instars of $S$.littoralis.

\begin{tabular}{|c|c|c|c|c|c|c|c|}
\hline $\begin{array}{l}\text { Treatment } \\
(\mathrm{ppm})\end{array}$ & $\begin{array}{l}\text { Larval } \\
\text { stages }\end{array}$ & $\begin{array}{c}\% \\
\text { Larval } \\
\text { Mortality }\end{array}$ & $\begin{array}{c}\text { Larval } \\
\text { Duration } \\
\text { (days) } \\
\text { mean } \pm \text { S. } \\
\text { E. }\end{array}$ & $\begin{array}{c}\% \\
\text { Pupal } \\
\text { Mortality }\end{array}$ & $\begin{array}{c}\text { Pupal } \\
\text { Duration } \\
\text { (days) } \\
\text { mean } \pm \text { S.E }\end{array}$ & $\begin{array}{c}\% \\
\text { Emerged } \\
\text { Moths }\end{array}$ & $\begin{array}{c}\text { Fecundity } \\
\text { no. of } \\
\text { eggs/female } \\
\text { Mean } \pm \text { S.E. }\end{array}$ \\
\hline Control & \multirow{7}{*}{$\begin{array}{c}2^{\text {nd }} \\
\text { instar } \\
\text { larvae }\end{array}$} & - & $\begin{array}{c}16 \\
( \pm 0.21)\end{array}$ & - & $\begin{array}{c}9.8 \\
( \pm 0 . .22)\end{array}$ & 99.8 & $\begin{array}{c}2499.1 \\
( \pm 319.78)\end{array}$ \\
\hline 0.125 & & 15 & $\begin{array}{c}12.5 \\
( \pm 0.84)\end{array}$ & 4 & $\begin{array}{c}7.5 \\
( \pm 0.92)\end{array}$ & 86 & $\begin{array}{c}500 \\
( \pm 41.23) \\
\end{array}$ \\
\hline 0.25 & & 35 & $\begin{array}{c}12.42 \\
( \pm 0.72)\end{array}$ & 6 & $\begin{array}{c}7.34 \\
( \pm 0.88)\end{array}$ & 64 & $\begin{array}{c}420 \\
( \pm 56.57)\end{array}$ \\
\hline 0.5 & & 45 & $\begin{array}{c}12.31 \\
( \pm 0.66)\end{array}$ & 8 & $\begin{array}{c}7.39 \\
( \pm 0.86) \\
\end{array}$ & 52 & $\begin{array}{c}380 \\
( \pm 56.16) \\
\end{array}$ \\
\hline 1 & & 54 & $\begin{array}{c}12.27 \\
( \pm 0.35)\end{array}$ & 10 & $\begin{array}{c}7.37 \\
( \pm 0.83)\end{array}$ & 42 & $\begin{array}{c}220 \\
( \pm 28.22)\end{array}$ \\
\hline 2 & & 60 & $\begin{array}{c}12.24 \\
( \pm 0.21) \\
\end{array}$ & 10 & $\begin{array}{c}7.25 \\
( \pm 0.82) \\
\end{array}$ & 36 & $\begin{array}{c}170 \\
( \pm 30.82)\end{array}$ \\
\hline 4 & & 82 & $\begin{array}{c}12.32 \\
( \pm 0.11)\end{array}$ & 15 & $\begin{array}{c}7.14 \\
( \pm 0.81)\end{array}$ & 15 & $\begin{array}{c}140 \\
( \pm 37.42)\end{array}$ \\
\hline Control & \multirow{7}{*}{$\begin{array}{c}4^{\text {th }} \\
\text { instar } \\
\text { larvae }\end{array}$} & - & 6 & - & $\begin{array}{c}8.5 \\
( \pm 0.88) \\
\end{array}$ & 100 & $\begin{array}{c}2254.2 \\
( \pm 319.78)\end{array}$ \\
\hline 0.125 & & 9.5 & $\begin{array}{c}5.5 \\
( \pm 4.5) \\
\end{array}$ & - & $\begin{array}{c}8.75 \\
( \pm 0.58) \\
\end{array}$ & 86 & $\begin{array}{c}1000 \\
( \pm 42.25)\end{array}$ \\
\hline 0.25 & & 13 & $\begin{array}{c}5 \\
( \pm 3)\end{array}$ & 2 & $\begin{array}{c}8.22 \\
( \pm 0.56) \\
\end{array}$ & 82 & $\begin{array}{c}925 \\
( \pm 62.1)\end{array}$ \\
\hline 0.5 & & 15.5 & $\begin{array}{c}4.8 \\
( \pm 1.5) \\
\end{array}$ & 4 & $\begin{array}{c}7.61 \\
( \pm 0.47) \\
\end{array}$ & 80 & $\begin{array}{c}860 \\
( \pm 52.27) \\
\end{array}$ \\
\hline 1 & & 16.5 & $\begin{array}{c}4.66 \\
( \pm 1.34) \\
\end{array}$ & 7 & $\begin{array}{c}7.5 \\
( \pm 0.45) \\
\end{array}$ & 79 & $\begin{array}{c}740 \\
( \pm 29.37) \\
\end{array}$ \\
\hline 2 & & 20 & $\begin{array}{c}4.33 \\
( \pm 3.67) \\
\end{array}$ & 9 & $\begin{array}{c}7.3 \\
( \pm 0.43) \\
\end{array}$ & 78 & $\begin{array}{c}620 \\
( \pm 8.17) \\
\end{array}$ \\
\hline 4 & & 27.5 & $\begin{array}{l}4.72 \\
( \pm 2)\end{array}$ & 10 & $\begin{array}{c}7.11 \\
( \pm 0.42) \\
\end{array}$ & 69 & $\begin{array}{c}530 \\
( \pm 21.6) \\
\end{array}$ \\
\hline
\end{tabular}

According to the data in Table 2, it appeared that a significant prolongation of larval duration was noticed at higher concentrations with mixture of Buxus chinensis, $B$. thuringiensis and nuclear polyhedrosis virus (NPV), where it was one day for $2^{\text {nd }}$ instars and 5 days for te $4^{\text {th }}$ instars, compared to 16 and 6 days for the control larvae. In case of pupal stage, which resulted from treated $4^{\text {th }}$ instar larvae of Spodoptera littoralis with mixture of Buxus chinensis, $B$. thuringiensis and nuclear polyhedrosis virus (NPV), it was effective on the mean percentage of pupation , mortality, deformed pupae and the pupal duration. 


\section{3- Effect of mixture of $B$. thuringiensis and nuclear polyhedrosis virus (NPV).}

When the $2^{\text {nd }}$ instars of Spodoptera littoralis were fed on castor oil leaves treated with mixture of $B$. thuringiensis and nuclear polyhedrosis virus (NPV), the different developmental stages were affected (Table 3). It was observed that the mean percentage of larval mortality were increased by increasing concentrations, where the higher mortality (62.5\%) was obtained at higher concentration for $2^{\text {nd }}$ instars but it was $54 \%$ for the $4^{\text {th }}$ instar larvae occurred at higher concentration, compared to no mortality for control larvae.

According to the data in Table (3) it was noticed a significant decrease in larval duration was achieved compared to 16 days for control larvae.

Table 2. The effect of Buxus chinensis, Dipel $2 X$ and nuclear polyhedrosis virus

\begin{tabular}{|c|c|c|c|c|c|c|c|}
\hline & V) $\mathrm{C}$ & $2^{\text {nd }}$ and & instar of & littora & & & \\
\hline $\begin{array}{l}\text { Treatment } \\
\text { (ppm) }\end{array}$ & $\begin{array}{l}\text { Larval } \\
\text { stages }\end{array}$ & $\begin{array}{c}\% \\
\text { Larval } \\
\text { Mortality }\end{array}$ & $\begin{array}{c}\text { Larval } \\
\text { Duration } \\
\text { (days) } \\
\text { mean } \pm \text { S.E. }\end{array}$ & $\begin{array}{c}\% \\
\text { Pupal } \\
\text { Mortality }\end{array}$ & $\begin{array}{c}\text { Pupal } \\
\text { Duration } \\
\text { (days) } \\
\text { mean } \pm \text { S.E. }\end{array}$ & $\begin{array}{c}\% \\
\text { Emerged } \\
\text { Moths }\end{array}$ & $\begin{array}{c}\text { Fecundity } \\
\text { no. of } \\
\text { eggs/female } \\
\text { Mean } \pm \text { S.E. }\end{array}$ \\
\hline Control & \multirow{6}{*}{$\begin{array}{c}2^{\text {nd }} \\
\text { instar } \\
\text { larvae }\end{array}$} & 0 & $\begin{array}{c}16 \\
( \pm 0.21)\end{array}$ & - & $\begin{array}{c}9.8 \\
( \pm 0 . .22)\end{array}$ & 100 & $\begin{array}{c}2499.1 \\
( \pm 319.78)\end{array}$ \\
\hline $\begin{array}{c}A+B+C \\
\% 100 \\
\end{array}$ & & 99 & $\begin{array}{c}1 \\
( \pm 0.01) \\
\end{array}$ & 1 & - & - & - \\
\hline $\begin{array}{c}A+B+C \\
\% 75\end{array}$ & & 90 & $\begin{array}{c}7 \\
( \pm 1) \\
\end{array}$ & 10 & $\begin{array}{c}5 \\
( \pm 0.1) \\
\end{array}$ & - & - \\
\hline $\begin{array}{c}A+B+C \\
\% 50\end{array}$ & & 85 & $\begin{array}{c}8 \\
( \pm 0.2) \\
\end{array}$ & 15 & $\begin{array}{c}6 \\
( \pm 0.1)\end{array}$ & - & - \\
\hline $\begin{array}{c}A+B+C \\
\% 25\end{array}$ & & 80 & $\begin{array}{c}9 \\
( \pm 0.13)\end{array}$ & 20 & $\begin{array}{c}7 \\
( \pm 0.1) \\
\end{array}$ & - & - \\
\hline $\begin{array}{c}A+B+C \\
\% 12.5 \\
\end{array}$ & & 70 & $\begin{array}{c}9.01 \\
( \pm 0.15) \\
\end{array}$ & 20 & $\begin{array}{c}7.2 \\
( \pm 0.5) \\
\end{array}$ & 10 & - \\
\hline Control & \multirow{6}{*}{$\begin{array}{c}4^{\text {th }} \\
\text { instar } \\
\text { larvae }\end{array}$} & 0 & $\begin{array}{c}16 \\
( \pm 0.21) \\
\end{array}$ & - & $\begin{array}{c}9.8 \\
( \pm 0.22) \\
\end{array}$ & 100 & $\begin{array}{c}2313 \\
( \pm 21.6) \\
\end{array}$ \\
\hline $\begin{array}{c}A+B+C \\
\% 100\end{array}$ & & 51 & $\begin{array}{c}5 \\
( \pm 1.87) \\
\end{array}$ & 6 & $\begin{array}{c}7 \\
( \pm 0.1)\end{array}$ & 43 & $\begin{array}{c}130 \\
( \pm 21.6)\end{array}$ \\
\hline $\begin{array}{c}A+B+C \\
\% 75\end{array}$ & & 45 & $\begin{array}{c}5.08 \\
( \pm 16.5)\end{array}$ & 8 & $\begin{array}{c}7.3 \\
( \pm 0.1)\end{array}$ & 47 & $\begin{array}{c}420 \\
( \pm 36.33) \\
\end{array}$ \\
\hline $\begin{array}{c}A+B+C \\
\% 50 \\
\end{array}$ & & 40 & $\begin{array}{c}5.1 \\
( \pm 1.83) \\
\end{array}$ & 8 & $\begin{array}{c}7.36 \\
( \pm 0.3) \\
\end{array}$ & 52 & $\begin{array}{c}585 \\
( \pm 142.83) \\
\end{array}$ \\
\hline $\begin{array}{c}A+B+C \\
\% 25 \\
\end{array}$ & & 35 & $\begin{array}{c}5.28 \\
( \pm 0.82) \\
\end{array}$ & 9 & $\begin{array}{c}7.41 \\
( \pm 1.1)\end{array}$ & 58 & $\begin{array}{c}725 \\
( \pm 20) \\
\end{array}$ \\
\hline $\begin{array}{l}A+B+C \\
\% 12.5\end{array}$ & & 30 & $\begin{array}{c}5.33 \\
( \pm 1.63)\end{array}$ & 10 & $\begin{array}{c}7.47 \\
( \pm 2.9)\end{array}$ & 60 & $\begin{array}{c}780 \\
( \pm .37)\end{array}$ \\
\hline
\end{tabular}

A: (NPV) $5 \times 10^{12} \mathrm{Pib} /$ larvae

B: B.thuringiensis (Diple 2X) IU

C: Buxus chinensis, $4 \mathrm{ppm}$

F values $=2267 \quad * * *$ L.S.D $0.05=1.963$ 
From table (3), the mean percentage pupal mortality was increased by increasing the concentrations. The higher pupal mortality (10\%) was obtained at higher concentration for the $2^{\text {nd }}$ instars but it was $(9 \%)$ for the $4^{\text {th }}$ instars occurred at higher concentration, compared to no pupal mortality for control larvae.

In case of adult stage, resulted from treated $2^{\text {nd }}$ and $4^{\text {th }}$ instars of $S$. littoralis, the mean percentage of emerged moths, fecundity and hatchability were greatly affected, as shown in Table 3. The mean percentage of emerged moths was decreased to $21.5 \%$ for $2^{\text {nd }}$ instars but it was $33 \%$ for the $4^{\text {th }}$ instars, as compared to $100 \%$ emerged moth for control.

From the results tabulated in tables (1-3) it could be concluded that the mixture of Buxus chinensis extract, $B$. thuringiensis and nuclear polyhedrosis virus (NPV) was the most active as natural pesticide against the $2^{\text {nd }}$ and the $4^{\text {th }}$ instar larvae of $S$. littoralis, followed by the mixture of $B$. thuringiensis and nuclear polyhedrosis virus (NPV), while Buxus chinensis extract alone had a slight effect in this respect. On the other hand, the pupation percentages and adult emergence averages were greatly affected. These results are similar to those obtained by many authors such Guirguis et al., (1991b), who reported that citrus oils revealed varying toxicities against cotton leafworm eggs, while it could be used as insecticide synergists against $S$. littoralis larvae. Also, Guirguis et al.,(1991) a found that the larval treatments with sublethal doses of ethyl acetate extract of $S$. fruticosa induced serious effects on the biology and biotic potential of S. littoralis. Eid et al., 1992 found that injection of $S$. littoralis larvae in the laboratory with sublethal doses of the Lemina ninor extract caused malformations in subsequent life stages. 
Table 3. Effect of B.thuringiensis (Diple 2X) and nuclear polyhedrosis virus (NPV) on $2^{\text {nd }}$ and $4^{\text {th }}$ instar of $S$. littoralis.

\begin{tabular}{|c|c|c|c|c|c|c|c|}
\hline Treatments & $\begin{array}{l}\text { Larval } \\
\text { stage }\end{array}$ & $\begin{array}{c}\% \\
\text { Larval } \\
\text { Mortality }\end{array}$ & $\begin{array}{c}\text { Larval } \\
\text { Duration } \\
\text { (days) } \\
\text { mean } \pm \text { S.E } \\
.\end{array}$ & $\begin{array}{c}\% \\
\text { Pupal } \\
\text { Mortality }\end{array}$ & $\begin{array}{c}\text { Pupal } \\
\text { Duration } \\
\text { (days) } \\
\text { mean } \pm \text { S.E } \\
\text {. }\end{array}$ & $\begin{array}{c}\% \\
\text { Emerge } \\
d \\
\text { Moths }\end{array}$ & $\begin{array}{c}\text { Fecundity } \\
\text { no. of } \\
\text { eggs/fem } \\
\text { ale } \\
\text { Mean } \pm \text { S.E }\end{array}$ \\
\hline$A+B(100 \%)$ & \multirow{6}{*}{$\begin{array}{c}2^{\text {nd }} \\
\text { instar } \\
\text { larvae }\end{array}$} & 62.5 & $\begin{array}{c}10.5 \\
( \pm 0.3)\end{array}$ & 6 & $\begin{array}{c}7.05 \\
( \pm 0.81)\end{array}$ & 68 & $\begin{array}{c}316 \\
( \pm 33.74)\end{array}$ \\
\hline$A+B(75 \%)$ & & 40 & $\begin{array}{c}10.55 \\
( \pm 1.1)\end{array}$ & 6 & $\begin{array}{c}7.25 \\
( \pm 0.81)\end{array}$ & 60 & $\begin{array}{c}426 \\
( \pm 88.72)\end{array}$ \\
\hline$A+B(50 \%)$ & & 32 & $\begin{array}{c}10.63 \\
( \pm 2.3)\end{array}$ & 8 & $\begin{array}{c}7.27 \\
( \pm 0.83) \\
\end{array}$ & 54 & $\begin{array}{c}537 \\
( \pm 121)\end{array}$ \\
\hline$A+B(25 \%)$ & & 22 & $\begin{array}{c}10.67 \\
( \pm 1.9)\end{array}$ & 10 & $\begin{array}{c}7.36 \\
( \pm 0.84)\end{array}$ & 34 & $\begin{array}{c}675 \\
( \pm 60.2)\end{array}$ \\
\hline$A+B(12.5 \%)$ & & 20 & $\begin{array}{c}10.75 \\
( \pm 3.1)\end{array}$ & 10 & $\begin{array}{c}7.42 \\
( \pm 0.86)\end{array}$ & 21.5 & $\begin{array}{c}980 \\
( \pm 46.2)\end{array}$ \\
\hline Control & & 0 & $\begin{array}{c}16 \\
( \pm 0.21)\end{array}$ & - & $\begin{array}{c}9.8 \\
( \pm 0.22)\end{array}$ & 100 & $\begin{array}{c}2499 \\
( \pm 319.8)\end{array}$ \\
\hline$A+B(100 \%)$ & \multirow{6}{*}{$\begin{array}{c}4^{\text {th }} \\
\text { instar } \\
\text { larvae }\end{array}$} & 54 & $\begin{array}{c}6.52 \\
( \pm 0.3)\end{array}$ & 5 & $\begin{array}{c}6.17 \\
( \pm 0.81)\end{array}$ & 71 & $\begin{array}{c}516 \\
( \pm 30.77)\end{array}$ \\
\hline$A+B(75 \%)$ & & 40 & $\begin{array}{c}6.54 \\
( \pm 1.1)\end{array}$ & 6 & $\begin{array}{c}6.02 \\
( \pm 0.81)\end{array}$ & 61 & $\begin{array}{c}626 \\
( \pm 89.12)\end{array}$ \\
\hline$A+B(50 \%)$ & & 32 & $\begin{array}{c}6.63 \\
( \pm 2.3) \\
\end{array}$ & 8 & $\begin{array}{c}5.82 \\
( \pm 0.84)\end{array}$ & 54 & $\begin{array}{c}737 \\
( \pm 123) \\
\end{array}$ \\
\hline$A+B(25 \%)$ & & 23 & $\begin{array}{c}6.62 \\
( \pm 1.9)\end{array}$ & 8 & $\begin{array}{c}5.80 \\
( \pm 0.86)\end{array}$ & 41 & $\begin{array}{c}875 \\
( \pm 62.1)\end{array}$ \\
\hline$A+B(12.5 \%)$ & & 7 & $\begin{array}{c}6.71 \\
( \pm 3.1) \\
\end{array}$ & 9 & $\begin{array}{c}5.64 \\
( \pm 0.86) \\
\end{array}$ & 33 & $\begin{array}{c}980 \\
( \pm 48.23)\end{array}$ \\
\hline Control & & 0 & $\begin{array}{c}16 \\
( \pm 0.21)\end{array}$ & - & $\begin{array}{c}8.29 \\
( \pm 0.23)\end{array}$ & 100 & $\begin{array}{c}2254 \\
( \pm 318.76)\end{array}$ \\
\hline
\end{tabular}
A: (NPV) $5 \times 10^{12} \mathrm{Pib} /$ larvae
B: B.thuringiensis (Diple 2X) IU
F values $=2267 \quad * * *$ L.S.D $0.05=1.963$

\section{REFERENCES}

1. Abbott, W. S. 1925. A method of computing the effectiveness of an insecticide. J.Econ.Entomol. 18, 265-267.

2. Abd El-Ghany, M. A., M. Farag, H. Yousef, M. H.M.Ahmed and A. H. Abd ElRahman. 2012. Insecticidal activity of Melia azedarach, Triterpeniods against Spodoptera littoralis (Boisd). J. American Sci. , 8(3): 661-667.

3. Abo El-Ghar, E. S. 1994. Influence of abamection and juvenile hormone analogues on food utilization, ingestion and larval growth of $S$. littoralis (Boisd).Bull. Ent. Soc. Egypt Econ. Ser. 20: 173-184. 
4. Antonious, A. G. and A. Rizk. 1994. Response of S. littoralis (Boisd) to neem Azadirachta indica ( $A$. juss) seeds oil Egypt. J. Appl. Sci. 9 (10): 424-434.

5. Chanda, S. and S. Chakravory. 1993. Food with neem oil affects life and development of rice moth, Corycyra cephalonica (Stainton) (Lepidoptera:Pyralidae). Entomol. 23(2):153-156.

6. Eid, M.A. , M.A. Kandil, E. B. Mori and G. E. Sayed. 1992. Bioassay of duck weed vegetation extracts. Insect Science and its Application, 13(5), 741-748.

7. El-Defrawy, M. E., A. Toppozada ,N. A. Mansour and M.Zeid 1964. Toxocological studies on the Egyptian cotton leafworm, Prodina litura I. Susceptibility of different larval instars to insecticides. J.Econ. Entomol. 57: 591-593.

8. Finney, D. J. 1971. Probit analysis ( $3^{\text {rd }}$ Cambridge University Press, New York. 333pp.).

9. Guirguis, M. W., K. M. Gouhar, W. M. Watson and R. M. Solim. 1991a. toxicity and latent effect of two plant extracts on the cotton leafworm Spodoptera littoralis (Boisd). Egypt J.Agric. Res. 69(1): 7-15.

10. Guirguis, M. W., K. M. Gouhar, W. M. Watson and R. M. Solim. 1991b. The toxic action of volatile oils extracted from Citruc fruit peels against different insect pests. Egypt J. Agric. Res., 69(1):15-21.

11. Ismail, L.A., N.A.Farag and M.A.Ragaei. 1999. Evaluation of certain insecticides and plant extracts on Bemisia tabaci Genn and their side effects on some natural enemies. J. Egypt Ger. Soc. Zool. Entomol. 2, 117-124.

12. Leora Software. 1994. POLO-PC Probit and Logit analysis. Leora Software, Barkley. CA.

13. Marei,S.S., E.M.Amr and N.Y.Salem. 2009. Effect of some plant oils on biological, physiological and biochemical aspects of Spodoptera littoralis (Boisd). Res. J. Agric. and Biol. Sci. 5(1): 103-107.

14. Nakanishi, K. 1977. Insect growth regulators from plants, natural products and the protection of the plants. (ed. Marini Bettolo, G.B.) Elsevier, Amsterdam.

15. Schmidt, G.H., A.A.Ahmed and M. Brewer. 1997. Effect of the Melia ozadarach extract on larval development and reproduction parameters of $S$. littoralis and Agrotis ipsilon. J.Econ. Entomol. 124,43-49.

16. Vagras,I. and F. Sehnal. 1973. USE OF jha's against Hyphantris cunea. Entomol. Exp. Appl., 16:115-122. 
تأثثر مخلوط مستخلص نبات الجوجوبا Buxus chinensis مع الدايبل 2x و الفيروس النووي NPV على بعض المظاهر الحيوية لحشرة دودة ورق القطن

صبحي محمود عبد الظاهر و عبيز محمود محمد

$$
\text { معهر بحوث وقابة النباتات - مركز البحوث الزراعية - الدقي - الجبزة }
$$

2x أجريت هذه الدراسة على تأثير كل من المستخلص النباتي لأوراق نبات الجوجوبا مع الداييل و الفيروس النووي NPV و مخلوط الداييل 2x و الفيروس النووي NPV على بيولوجية دودة ورق القطن . و قد أوضحت النتائج أنه بمكن تقسيم المواد تبعا لثدة تأثبرها على الحشرة الى ثلى ثلاث

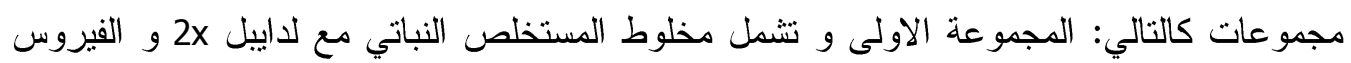
النووي ذات تأثير قوي. المجموعة الثانية مخلوط الداييل 2x و الفيروس النووي ذات نأثير منوسط و المجموعة الثالثة و تشمل المستخلص النباتي الضعيفة التأثير 